\title{
A computer-assisted proof of symbolic dynamics in Hyperion's rotation
}

\author{
Anna Gierzkiewicz ${ }^{1}$ (D) Piotr Zgliczyński ${ }^{2}$
}

Received: 2 February 2019 / Revised: 6 June 2019 / Accepted: 15 June 2019 / Published online: 2 July 2019

(c) The Author(s) 2019

\begin{abstract}
Hyperion is a moon of Saturn, known of its non-round shape. Its rotation is often modelled by equations of motion of an ellipsoidal satellite. The model is expected to be chaotic for a large range of parameters. The paper contains a rigorous proof of the existence of symbolic dynamics in the model. In other words, there exist infinitely many periodic orbits of arbitrary periods coded by the sequences of two symbols. The proofs are computer assisted, based on the interval arithmetic by the use of CAPD C++ library.
\end{abstract}

Keywords Computer-assisted proof · Hyperion · Symbolic dynamics · Interval Newton method

Mathematics Subject Classification 37D45 · 37N05 · 70F15

\section{Introduction}

A motivation of our study was the broadly known example of chaotic motion in the Solar System, i.e. the tumbling of Hyperion, one of the Saturn's moons. The shape of Hyperion significantly differs from spherical: it is roughly an ellipsoid with the rate of principal moments of inertia $\frac{\Theta_{2}-\Theta_{1}}{\Theta_{3}} \approx 0.26$, where $\Theta_{3}>\Theta_{2}>\Theta_{1}$. Hyperion's Keplerian elliptic orbit (long semi-axis $a=1,500,933 \mathrm{~km}$, period $T=21.276 \mathrm{~d}$ ) is non-circular with eccentricity $e \approx 0.1$ (Jay Klavetter 1989).

The analysis of Voyager and Voyager 2 observational data could not fit Hyperion's rotation into any certain period, which was the base of supposition that its tumbling may be chaotic.

Work of A.G. and P.Z. was supported by National Science Center (NCN) of Poland under Project No. 2015/19/B/ST1/01454.

$\bowtie \quad$ Anna Gierzkiewicz

anna.gierzkiewicz@urk.edu.pl

Piotr Zgliczyński

umzglicz@cyf-kr.edu.pl

1 Department of Applied Mathematics, University of Agriculture in Kraków, ul. Balicka 253c, 30-198 Kraków, Poland

2 Institute of Computer Science, Jagiellonian University, ul. Łojasiewicza 6, 30-348 Kraków, Poland 
The natural attempt (Wisdom et al. 1984) to explain this phenomenon was to compare it to a classical Danby (1962) model of rotation of an oblate satellite, see (Greiner 2009, Example 27.5). The model assumes that the satellite is of ellipsoidal shape and orbits a massive distant body in a Keplerian ellipse orbit with significant eccentricity. It also states that the longest (or the shortest) ellipsoid's axis is always perpendicular to the orbit's plane, which is crucial to simplicity of the model: it implies one axis of rotation only. This last assumption is justified as the basic analysis of Euler equations of the rigid body motion shows that this 'normal' state is stable (Danby 1962).

The model does not, unfortunately, fit sufficiently: the key assumption on perpendicularity of the rotation axis to the orbit plane is not true in Hyperion's case. It is tumbling, and its rotation rate is more than 4 times too fast for Hyperion to be synchronous (Harbison et al. 2011). Nevertheless, the model is interesting and applicable in many other cases, like moon's or Mercury's libration. Proving the existence of chaos in it can also be helpful in the more general modelling of Hyperion's motion. This is the reason why in this paper we fix the parameters to Hyperion's case: $\omega=0.89 \pm 0.22$ and $e=0.1042$ (Jay Klavetter 1989).

The model with the above parameters was, as mentioned, explored in some articles, such as Black et al. (1995), Jay Klavetter (1989), Wisdom et al. (1984) and Tarnopolski (2015). The statement of chaotic rotation is based there on the picture of Poincaré section $S:\{f=0\}$, which visibly contains a large chaotic region (see also Fig. 2). The Lyapunov Characteristic Exponents were also numerically calculated. The rigorous proof of chaoticity would set the mathematical ground to their theses and also can present an elegant application for the topological methods combined with rigorous numerics (CAPD Group 2017). In this paper, we understand the existence of chaos in a dynamical system as the semi-conjugacy of its dynamics onto the shift dynamics on the space of bi-infinite sequences of two symbols. Such a phenomenon is known in the literature as symbolic dynamics (Morse and Hedlund 1938; Moser 1973). It means, in particular, the existence of a compact invariant subset of the phase space with typical chaotic phenomena: periodic orbits of arbitrary period, dense orbit or sensitivity to the initial conditions.

The paper is organized as follows: in Sect. 2, we present the model, the system of ODEs and its basic properties. Sections 3 and 4 contain description of main topological tools used in our work. Section 5 presents our results for the symbolic dynamics in our model.

\section{The model}

\subsection{The equations}

We shortly recollect the derivation of the model (Danby 1962, Eq. 14.3.1).

An ellipsoidal satellite $S$ orbits a massive body $F$ in a Kepler ellipse orbit. Therefore, its true anomaly $f$ fulfils the equation

$$
f^{\prime}=\frac{(1+e \cos f)^{2}}{\left(1-e^{2}\right)^{3 / 2}} \text {. }
$$

Equation (1) has a symmetry: if $t \mapsto f(t)$ is a solution, then $t \mapsto-f(-t)$ is a solution. Also, solutions are strictly increasing.

The shortest axis of the satellite is perpendicular to the plane of the orbit. The rotation is expressed by the angle $\theta$ (see Fig. 1) between the longest axis of $S$ and the long axis of 
Fig. 1 Illustration of the model

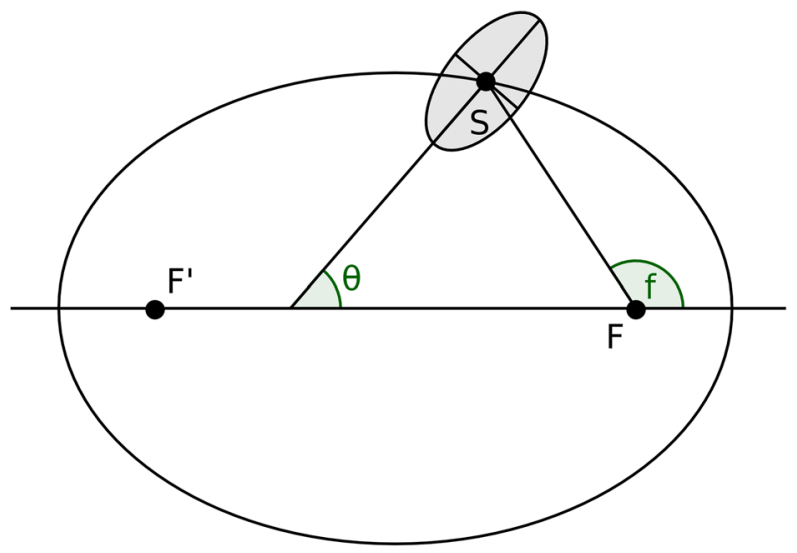

the orbit. Then $\theta$ fulfils the second-order ordinary differential equation (Greiner 2009, Eq. 27.97)

$$
\theta^{\prime \prime}=-\frac{\omega^{2}}{2 r^{3}} \sin 2(\theta-f), \quad \text { where } \quad r=\frac{1-e^{2}}{1+e \cos f} .
$$

The parameter

$$
\omega^{2}=\frac{\Theta_{2}-\Theta_{1}}{\Theta_{3}} \in[0,1]
$$

may be understood as a measure of oblateness of the satellite.

\subsection{The dynamical system}

In general, Eqs. (1) and (2) induce a three-dimensional dynamical system

$$
\left\{\begin{array}{l}
\theta^{\prime}=\phi \\
\phi^{\prime}=-\frac{\omega^{2}}{2 r^{3}} \sin 2(\theta-f) \\
f^{\prime}=\frac{(1+e \cos f)^{2}}{\left(1-e^{2}\right)^{3 / 2}}
\end{array}\right.
$$

with parameters $e$ and $\omega^{2}$. The rotation angle $\theta \in[0, \pi]$ and $f \in[0,2 \pi]$, so the phase space for the system (4) is $(\theta, \phi, f) \in \mathbb{R} / \pi \mathbb{Z} \times \mathbb{R} \times \mathbb{R} / 2 \pi \mathbb{Z}$.

\subsection{Poincaré map}

We study the Poincare map $P$ of the system (4) on the two-dimensional section $S:\{f=0\}$, i.e. the map

$$
P(\theta, \phi)=\Phi(T(\theta, \phi),(\theta, \phi, f=0)),
$$

where $\Phi$ is the dynamical system induced by (4) and $T=T(\theta, \phi)$ is a first recurrence time. Note that the domain of so-defined map is $\operatorname{Dom}_{P}=\mathbb{R}_{/ \pi \mathbb{Z}} \times \mathbb{R}$, because $f$ is strictly increasing and of bounded variation.

The main fragment of the Poincaré section $S$ with twelve orbits marked in different colours is depicted in Fig. 2. 


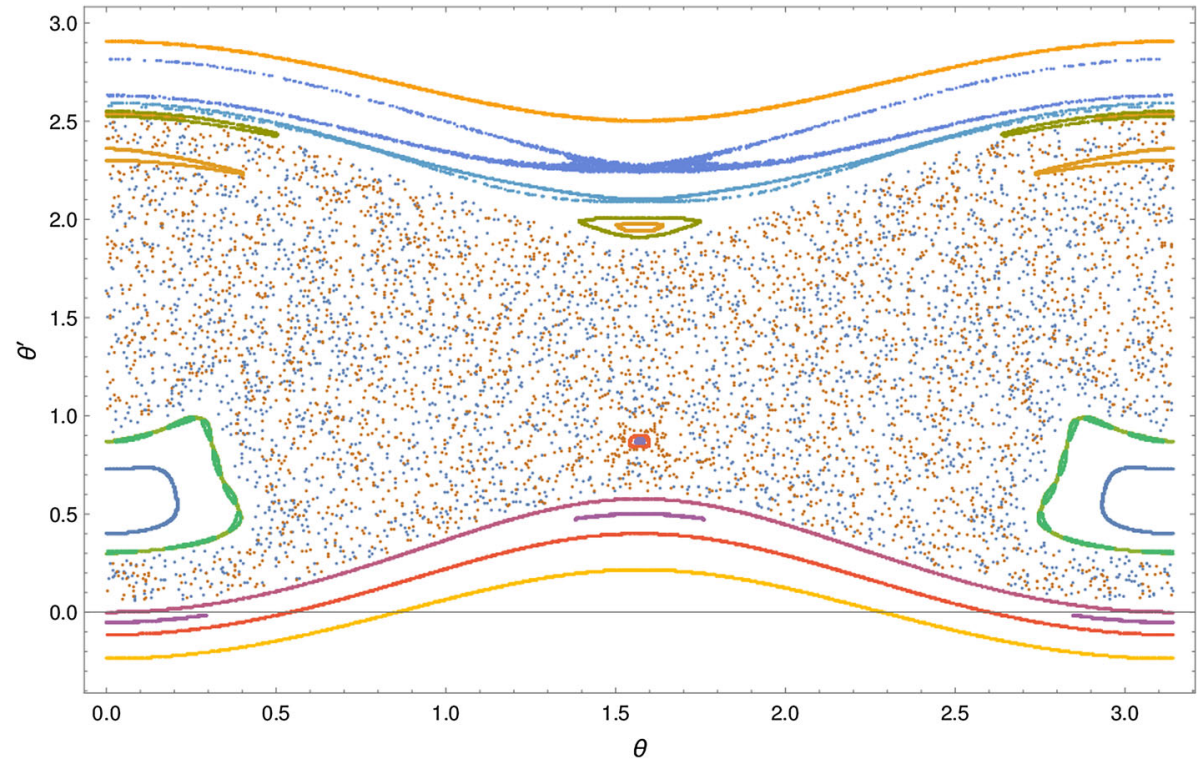

Fig. 2 Poincaré section $S:\{f=0\}, e=0.1, \omega^{2}=0.79$

What can be immediately noticed is a probable symmetry of the map $P$, because the section seems to have reflection symmetries with respect to $\theta=0=\pi$ and $\theta=\frac{\pi}{2}$ lines. Indeed, setting $t \mapsto-t$ to the equations we notice that if $(\theta(t), \phi(t), f(t))$ is a solution, then so is $(-\theta(-t), \phi(-t),-f(-t))$, and consequently,

$$
\pi_{\theta} P(\theta, \phi)=-\pi_{\theta} P^{-1}(-\theta, \phi), \quad \pi_{\phi} P(\theta, \phi)=\pi_{\phi} P^{-1}(-\theta, \phi) .
$$

Then, using the periodicity of the phase space: $-\theta=0-\theta=\pi-\theta$, we explain the two symmetries of the Poincaré section.

For further consideration, we denote the $\left\{\theta=\frac{\pi}{2}\right\}$-hyperplane reflectional time-reversing symmetry of the extended phase space by $R$, so

$$
R(\theta, \phi, f, t)=(\pi-\theta, \phi,-f,-t) .
$$

We will also, if it is understandable, denote by $R$ its restriction to $S: R(\theta, \phi)=(\pi-\theta, \phi)$.

\subsection{Chaos in the system (4)}

The other natural observation based on Fig. 2 is a large region of probable chaos for (more or less) $0<\phi=\theta^{\prime}<2.0$, with some elliptic islands. The chaotic behaviour in terms of stability and tidal evolution was studied by Wisdom et al. (1984). The Lyapunov Characteristic Exponents (LCE) occurred to be positive, which was the reason to treat Hyperion's motion as chaotic in the subsequent literature. LCE of the system with a wide range of $e$ and $\omega^{2}$ were also explored by Tarnopolski (2015). 


\section{Periodic orbits via topological covering}

Topological tools that we used in detecting periodic orbits for (4) were introduced in detail in Wilczak and Zgliczyński (2003) and Wilczak and Zgliczyński (2006). Here we recollect them shortly and present some intuition.

By $\mathbb{B}_{k}$, we will denote the $k$-dimensional unit ball, that is,

$$
\mathbb{B}_{k}=\left\{\mathbf{x} \in \mathbb{R}^{k}:\|\mathbf{x}\|<1\right\} .
$$

For a topological set $A \subset \mathbb{R}^{k}$, we will denote by $\bar{A}$, int $A$ and $\partial A$ its closure, interior and boundary, respectively.

\subsection{H-sets}

The basic notion is

Definition 1 (Wilczak and Zgliczyński 2003, Definition 3.1) An h-set is a quadruple $N=$ $\left(|N|, u(n), s(N), c_{N}\right)$, where $|N|$ is a compact subset of $\mathbb{R}^{n}$, which we will call a support of $a h$-set (or simply an $h$-set), and

1. two numbers $u(N)$ and $s(N) \in \mathbb{N} \cup\{0\}$ complement the dimension of space:

$$
u(N)+s(N)=n
$$

we will call them the exit and entry dimension, respectively;

2. the homeomorphism $c_{N}: \mathbb{R}^{n} \rightarrow \mathbb{R}^{n}=\mathbb{R}^{u(N)} \times \mathbb{R}^{s(N)}$ is such that

$$
c_{N}(|N|)=\overline{\mathbb{B}_{u(N)}} \times \overline{\mathbb{B}_{S(N)}},
$$

where $\overline{\mathbb{B}_{k}}$ denotes a closed unit ball of dimension $k$.

We set also some useful notions:

$$
\begin{aligned}
\operatorname{dim} N & =n, \\
N_{\mathrm{c}} & =\overline{\mathbb{B}_{u(N)}} \times \overline{\mathbb{B}_{s(N)}}, \\
N_{\mathrm{c}}^{-} & =\partial \mathbb{B}_{u(N)} \times \overline{\mathbb{B}_{s(N)}}, \\
N_{\mathrm{c}}^{+} & =\overline{\mathbb{B}_{u(N)}} \times \partial \mathbb{B}_{s(N)}, \\
N^{-} & =c_{N}^{-1}\left(N_{\mathrm{c}}^{-}\right), \quad N^{+}=c_{N}^{-1}\left(N_{\mathrm{c}}^{+}\right) .
\end{aligned}
$$

As one can notice, the notions with the subscript $\mathrm{c}$ refer to the 'straight' coordinate system in the image of $c_{N}$. The last two sets $N^{-}$and $N^{+}$defined above are often called the exit set and the entrance set, respectively.

Therefore, we can assume that an $h$-set is a product of two unitary balls moved to some coordinate system with the exit and entrance sets distinguished.

\subsection{Covering and back-covering}

We define the notion of topological covering:

Definition 2 (Wilczak and Zgliczyński 2003, Definition 3.4, simplified) Let $f:|N| \rightarrow \mathbb{R}^{n}$ be a continuous map and two $h$-sets $M$ and $N$ are such that $u(M)=u(N)=u$ and 

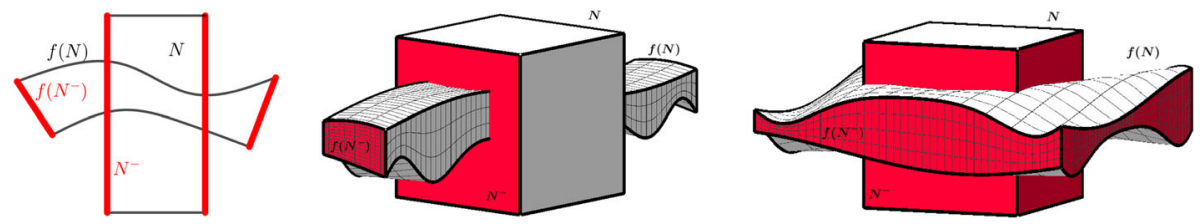

Fig. 3 Examples of topological self-covering $N \stackrel{f}{\Longrightarrow} N$ in $\mathbb{R}^{2}$ (left) and $\mathbb{R}^{3}$ : with one exit direction (middle) and two exit directions (right). The exit sets and their images are marked in red

$s(M)=s(N)=s$. Denote $f_{\mathrm{c}}=c_{N} \circ f \circ c_{M}^{-1}: M_{\mathrm{c}} \rightarrow \mathbb{R}^{u} \times \mathbb{R}^{s}$. We say that that $M$ $f$-covers the $h$-set $N$, if

1. there exists a continuous homotopy $h:[0,1] \times M_{\mathrm{c}} \rightarrow \mathbb{R}^{u} \times \mathbb{R}^{s}$, such that

$$
\begin{array}{rlrl}
h_{0} & =f_{\mathrm{c}}, & \\
h\left([0,1], M_{\mathrm{c}}^{-}\right) \cap N_{\mathrm{c}} & =\varnothing & & \text { (the exit condition), } \\
h\left([0,1], M_{\mathrm{c}}\right) \cap N_{\mathrm{c}}^{+} & =\varnothing & \text { (the entry condition). }
\end{array}
$$

2. If $u>0$, then there exists a linear map $A: \mathbb{R}^{u} \rightarrow \mathbb{R}^{u}$ such that

$$
\begin{aligned}
& h_{1}(x, y)=(A(x), 0) \quad \text { for } x \in \overline{\mathbb{B}_{u}} \text { and } y \in \overline{\mathbb{B}_{s}}, \\
& A\left(\partial \mathbb{B}_{u}\right) \subset \mathbb{R}^{u} \backslash \mathbb{B}_{u} .
\end{aligned}
$$

If $M f$-covers $N$, we simply denote it by $M \stackrel{f}{\Longrightarrow} N$. See Fig. 3 for an illustration of covering in some low-dimensional cases.

Sometimes, it is more convenient to use the backward covering, which one may understand as the covering backwards in time.

Definition 3 If $N$ is an $h$-set, then we define an $h$-set $N^{\mathrm{T}}$ as

$-\left|N^{\mathrm{T}}\right|=|N|$;

$-u\left(N^{\mathrm{T}}\right)=s(N)$ and $s\left(N^{\mathrm{T}}\right)=u(N)$;

- $c_{N^{\mathrm{T}}}: \mathbb{R}^{n} \ni x \longmapsto j\left(c_{N}(x)\right) \in \mathbb{R}^{u\left(N^{\mathrm{T}}\right)} \times \mathbb{R}^{s\left(N^{\mathrm{T}}\right)}=\mathbb{R}^{n}$, where $j: \mathbb{R}^{u\left(N^{\mathrm{T}}\right)} \times \mathbb{R}^{s\left(N^{\mathrm{T}}\right)} \ni$ $(p, q) \longmapsto(q, p) \in \mathbb{R}^{s\left(N^{\mathrm{T}}\right)} \times \mathbb{R}^{u\left(N^{\mathrm{T}}\right)}$.

As we can see, the $h$-set $N^{\mathrm{T}}$ is just the $h$-set $N$ with the entrance and exit sets swapped.

Definition 4 Let $M$ and $N$ be two $h$-sets such that $u(M)=u(N)=u$ and $s(M)=s(N)=s$. Let $f: \operatorname{Dom}_{f} \subset \mathbb{R}^{n} \rightarrow \mathbb{R}^{n}$ be such that $f^{-1}:|N| \rightarrow \mathbb{R}^{n}$ is well defined and continuous.

Then we say that $M$ back-covers $N$ and denote by $M \stackrel{f}{=} N$, iff $N^{\mathrm{T}} \stackrel{f^{-1}}{\Longrightarrow} M^{\mathrm{T}}$.

If either $M \stackrel{f}{\Longleftarrow} N$ or $M \stackrel{f}{\Longleftrightarrow} N$, then we will write $M \stackrel{f}{\Longleftrightarrow} N$.

In general, if $N_{0} \stackrel{f}{\Longrightarrow} N_{1}$ and $N_{1} \stackrel{f}{\Longrightarrow} N_{2}$, then not necessarily $N_{0} \stackrel{f^{2}}{\Longrightarrow} N_{2}$, but covering has the property of tracking orbits. The basic application of topological covering is the following theorem, stating the existence of a periodic orbit related to a sequence of coverings.

Theorem 1 (Wilczak and Zgliczyński 2003, Theorem 3.6, simplified) Suppose there exists a sequence of h-sets $N_{0}, \ldots N_{n}=N_{0}$, such that

$$
N_{0} \stackrel{f}{\Longleftrightarrow} N_{1} \stackrel{f}{\Longleftrightarrow} \ldots \stackrel{f}{\Longleftrightarrow} N_{n}=N_{0} \text {, }
$$




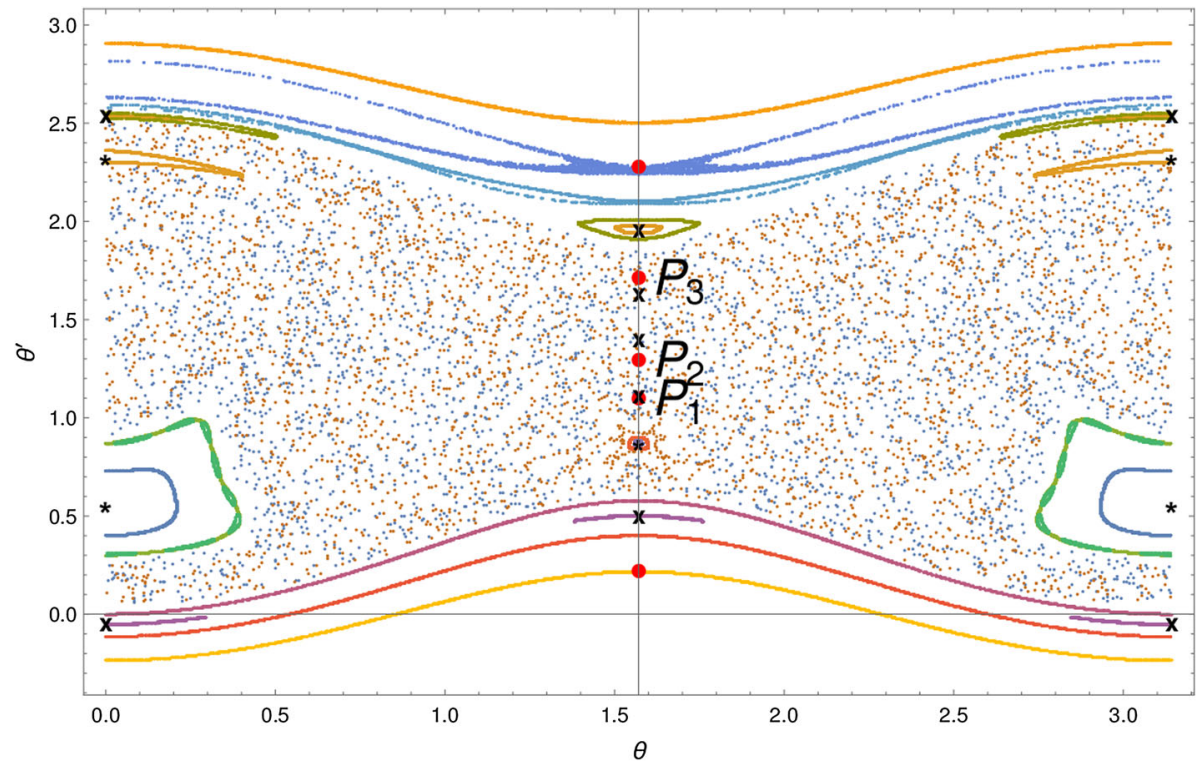

Fig. 4 Periodic points of $P$, found via interval Newton method. The points marked by red dots are hyperbolic; black asterisks ' $\star$ ' are elliptic. The points marked by black ' $\mathrm{X}$ ' are the stationary points for $P^{2}$

then there exists a point $x \in$ int $\left|N_{0}\right|$, such that $f^{k}(x) \in$ int $\left|N_{k}\right|$ for $k=0,1, \ldots, n$ and $f^{n}(x)=x$.

In particular, if $N_{0} \stackrel{f}{\Longrightarrow} N_{0}$, then in $N_{0}$ we have a stationary point for the map $f$. Note also that if the map is a Poincaré map $P$, then a stationary point for $P$ or $P^{k}$ lies on a periodic orbit for the dynamical system.

\subsection{Periodic orbits in Hyperion's rotation}

Using Theorem 1, we find some stationary points for $P$, i.e. periodic orbits for the system (4). Their existence is proved rigorously via the interval Newton method (Moore 1966; Neumaier 1991) implemented in C++ language with the use of CAPD library (CAPD Group 2017). Using this method, one can also estimate the eigenvalues of the derivative in the stationary point, so it is possible to prove rigorously whether the points are hyperbolic. Those periodic points are depicted on the Poincaré map $P$ in Fig. 4. Three of them, denoted as $P_{1}, P_{2}$ and $P_{3}$, will be important in further consideration.

The list of small intervals on the $\left\{\theta=\frac{\pi}{2}\right\}$ axis containing $P_{1}, P_{2}, P_{3}$ is shown in Table 1 . From now on, we will denote by $P_{1}, P_{2}, P_{3}$ the stationary points as well as the small sets containing them, described in this table.

The hyperbolic points $P_{1}, P_{2}, P_{3}$, presented in Fig. 4, can be also detected up to a small neighbourhood using covering relations. It is sufficient to find a self-covering compact set. This method, however, does not prove neither the uniqueness of the stationary point inside the set nor its hyperbolicity. The examples of the self-covering sets for $P_{1}, P_{2}, P_{3}$ are presented in Fig. 5. 
Table 1 Localization of three stationary points of $P$, found via interval Newton method

\begin{tabular}{ll}
\hline Point & Interval on $\left\{\theta=\frac{\pi}{2}\right\}$ \\
\hline$P_{1} \in$ & {$[1.099105177037064,1.099105177037082]=1.0991051770370_{64}^{82}$} \\
$P_{2} \in$ & {$[1.294219468594181,1.294219468594242]=1.294219468594_{181}^{242}$} \\
$P_{3} \in$ & {$[1.711370591161749,1.711370591161875]=1.711370591161_{749}^{875}$} \\
\hline
\end{tabular}
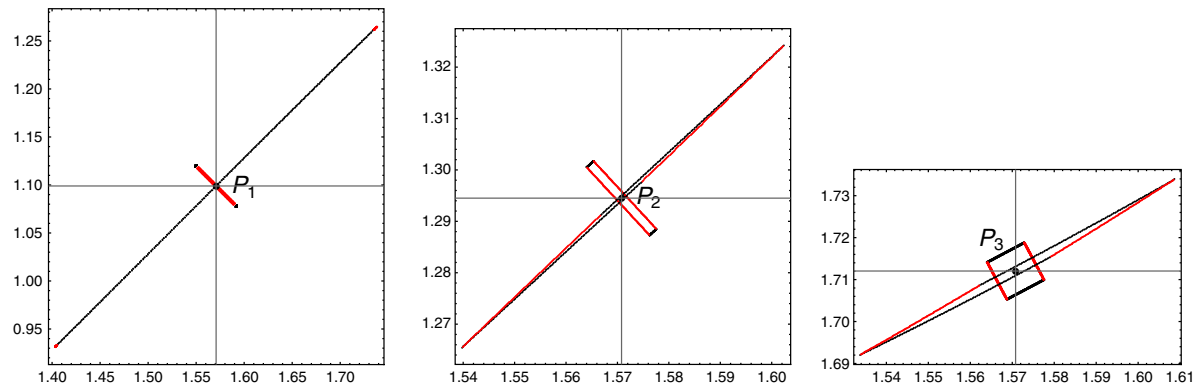

Fig. 5 Self-covering sets (the rectangles) proving existence of stationary points $P_{1}, P_{2}, P_{3}$. The exit sets and their images are marked in red

\section{Symbolic dynamics detecting via covering}

For the next study, we assume that the continuous map $f: \mathbb{R}^{2} \rightarrow \mathbb{R}^{2}$ and all $h$-sets $N_{i}$ contained in $\mathbb{R}^{2}$ have entry and exit dimensions equal to 1 , that is, $s\left(N_{i}\right)=u\left(N_{i}\right)=1$. Following the above assumptions, the notions related to a $h$-set get simpler, because the balls are just the closed intervals: $\overline{\mathbb{B}_{u(N)}}=\overline{\mathbb{B}_{S(N)}}=[-1,1]$ :

$-N_{\mathrm{c}}=[-1,1]^{2}$,

$-N_{\mathrm{c}}^{-}=\{-1,1\} \times[-1,1], \quad N_{\mathrm{c}}^{+}=[-1,1] \times\{-1,1\}$,

$-N^{-}$and $N^{+}$are topologically a sum of two disjoint intervals.

\subsection{Symbolic dynamics for a two-dimensional map}

A model chaotic behaviour for our purposes is the shift map on the set of bi-infinite sequences of two symbols, that is, the space $\Sigma_{2}=\{0,1\}^{\mathbb{Z}}$ as a compact metric space with the metric

$$
\text { for } c=\left\{c_{n}\right\}_{n \in \mathbb{Z}}, \quad c^{\prime}=\left\{c_{n}^{\prime}\right\}_{n \in \mathbb{Z}}, \quad \operatorname{dist}\left(c, c^{\prime}\right)=\sum_{n=-\infty}^{+\infty} \frac{\left|c_{n}-c_{n}^{\prime}\right|}{2^{|n|}},
$$

which induces the product topology. The shift map $\sigma: \Sigma_{2} \rightarrow \Sigma_{2}$, given by

$$
(\sigma(c))_{n}=c_{n+1},
$$

is a homeomorphism of $\Sigma_{2}$ with well-known chaotic properties like the existence of a dense orbit, existence of orbit of any given period or that the set of periodic orbits is dense in the whole space.

In our study, by the chaotic behaviour of a dynamical system we understand the existence of a compact set $I$ invariant for the Poincaré map $P$ (or sometimes its higher iteration) and a continuous surjection $g: I \rightarrow \Sigma_{2}$ such that $\left.P\right|_{I}$ is semi-conjugated to $\sigma$, that is, 
Fig. 6 A topological horseshoe: each $N_{0,1}$ covers itself and the other set. The exit sets of $N_{0}$ and $N_{1}$ are marked in red and green, respectively

$$
\begin{aligned}
& N_{0} \stackrel{f}{\Longrightarrow} N_{0}, \quad N_{0} \stackrel{f}{\Longrightarrow} N_{1}, \\
& N_{1} \stackrel{f}{\Longrightarrow} N_{0}, \quad N_{1} \stackrel{f}{\Longrightarrow} N_{1} .
\end{aligned}
$$

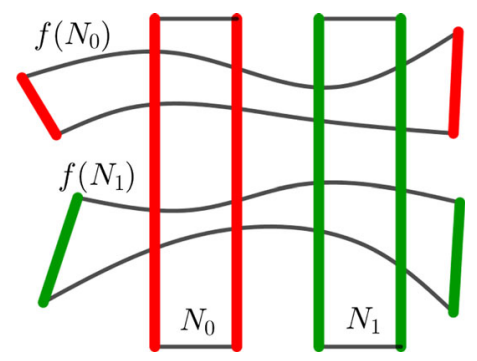

$$
\left.g \circ P\right|_{I}=\sigma \circ g .
$$

Then one may say that $P$ admits on $I$ at least as rich dynamics as $\sigma$ on $\Sigma_{2}$. The system $\left(\Sigma_{2}, \sigma\right)$ or any system (semi-)conjugated to it is sometimes described in the literature as symbolic dynamics (Morse and Hedlund 1938).

For better understanding the nature of symbolic dynamics, let us recall shortly that the discrete system $\left(\Sigma_{2}, \sigma\right)$ defined above shows all the typical chaotic phenomena as transitivity, density of periodic orbits or sensitivity to the initial conditions. There exists a periodic orbit of any prescribed period. The system $\left(\Sigma_{2}, \sigma\right)$ can be also imagined as a model of ideal randomness, obviously related to the probability space of an infinite experiment of coin tossing.

\subsection{Topological horseshoe}

A simple example of symbolic dynamics semi-conjugated to $\sigma$ is a horseshoe:

Definition 5 Let $N_{0}$ and $N_{1} \subset \mathbb{R}^{2}$ be two disjoint $h$-sets. We say that a continuous map $f: \mathbb{R}^{2} \rightarrow \mathbb{R}^{2}$ is a topological horseshoe for $N_{0}$ and $N_{1}$ if (see Fig. 6)

$$
\begin{aligned}
& N_{0} \stackrel{f}{\Longrightarrow} N_{0}, \quad N_{0} \stackrel{f}{\Longrightarrow} N_{1}, \\
& N_{1} \stackrel{f}{\Longrightarrow} N_{0}, \quad N_{1} \stackrel{f}{\Longrightarrow} N_{1} .
\end{aligned}
$$

It can be shown that for any topological horseshoe we obtain symbolic dynamics.

Theorem 2 (Zgliczyński and Gidea 2004, Theorem 18) Let $f$ be a topological horseshoe for $N_{0}$ and $N_{1}$. Denote by $I=\operatorname{Inv}\left(N_{0} \cup N_{1}\right)$ the invariant part of the set $N_{0} \cup N_{1}$ under $f$, and define a map $g: I \rightarrow \Sigma_{2}$ by

$$
g(x)_{k}=j \in\{0,1\} \quad \text { iff } \quad f^{k}(x) \in N_{j} .
$$

Then $g$ is a surjection satisfying $\left.g \circ f\right|_{I}=\sigma \circ g$, and therefore, $f$ is semi-conjugated to the shift map $\sigma$ on $\Sigma_{2}$.

The conjugacy with the model space $\Sigma_{2}$ may be understood as follows: for any sequence of the symbols 0 and 1, there exists an orbit of the discrete system generated by $f$ passing through the sets $N_{0}$ and $N_{1}$ in the order given by the sequence. Moreover, if the sequence is periodic, then so is the orbit.

Corollary 1 Let $f$ be a topological horseshoe for $N_{0}$ and $N_{1}$. Then it follows from Theorem 1 that for any finite sequence of zeros and ones $\left(a_{0}, a_{1}, \ldots, a_{n-1}\right), a_{i} \in\{0,1\}$, there exists $x \in N_{a_{0}}$ such that

$$
f^{i}(x) \in \operatorname{int} N_{a_{i}} \text { and } f^{n}(x)=x .
$$




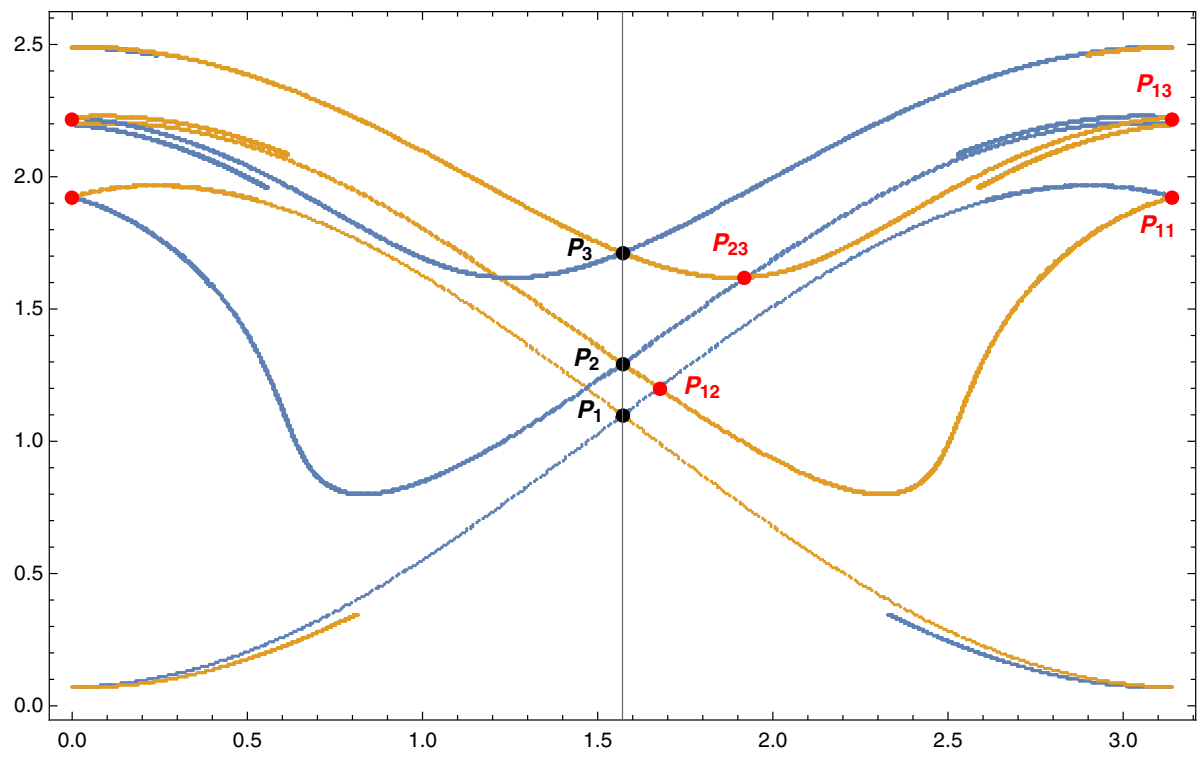

Fig. 7 Fragments of stable (orange) and unstable (blue) manifolds of stationary points $P_{1}, P_{2}, P_{3}$. Some points of intersections of the manifolds are marked in red, namely $P_{11}, P_{12}, P_{13}, P_{23}$

Remark 1 Note also that the following two chains of coverings or back-coverings:

$$
\begin{aligned}
& N_{0} \stackrel{f}{\Longleftrightarrow} N_{0} \stackrel{f}{\Longleftrightarrow} N_{1} \stackrel{f}{\Longleftrightarrow} \ldots \stackrel{f}{\Longleftrightarrow} N_{k}=M_{0}, \\
& M_{0} \stackrel{f}{\Longleftrightarrow} M_{0} \stackrel{f}{\Longleftrightarrow} M_{1} \stackrel{f}{\Longleftrightarrow} \ldots \stackrel{f}{\Longleftrightarrow} M_{k}=N_{0}
\end{aligned}
$$

indicate symbolic dynamics for $f^{k}$ in the sets $N_{0}$ and $M_{0}$, with the use of Theorem 1 .

\section{Symbolic dynamics in Hyperion's rotation system}

Numerical calculations suggest that the three hyperbolic points $P_{1}, P_{2}$ and $P_{3}$ detected above (see Table 1) have an interesting property: their stable and unstable manifolds intersect (for the same point and also pairwise), see Fig. 7. The intersections seem to be transversal, which is a clue for searching for the symbolic dynamics in the Poincare map $P$.

We found six topological horseshoes related to the intersections mentioned above. Below we present the $h$-sets and their covering relations. Similarly as in Wilczak and Zgliczyński (2003), the $h$-sets are parallelograms of the form $N=p+A \cdot b$, which are the base cubes $b$ transformed to some affine coordinate system, where

- $p$ are some small interval vectors containing base points (such as $P_{1}, P_{2}$ and $P_{3}$ );

- interval matrices $A$ are usually one of the eigenvectors matrices: 


$$
\begin{aligned}
& \mathbf{M}_{1}=\left[\begin{array}{ll}
0.7064565371_{793425}^{812066} & 0.7077564277_{852203}^{908148} \\
0.7064565371_{793425}^{812066} & -0.7077564277_{908146}^{852203}
\end{array}\right], \\
& \mathbf{M}_{2}=\left[\begin{array}{ll}
0.7338248323_{735296}^{818986} & 0.679338733_{8943307}^{9188653} \\
0.7338248323_{735297}^{818987} & -0.679338733_{9188652}^{8943306}
\end{array}\right], \\
& \mathbf{M}_{3}=\left[\begin{array}{ll}
0.8831685403_{716123}^{830619} & 0.4690557848_{18765}^{524839} \\
0.8831685403_{716135}^{830631} & -0.4690557848_{524816}^{187629}
\end{array}\right],
\end{aligned}
$$

or, in the case of connecting $P_{3}$ to itself, some matrices corrected to point directions of the dynamics on the stable or unstable manifolds.

\subsection{Outline of the computer-assisted proofs}

In the following six cases, we find some chains of $h$-sets $N$ which are rectangles transformed to some affine coordinate systems (parallelograms on the section $S$ ). The computer-assisted proof finds their overestimated images through the Poincare map $P$ and encloses them in rectangles (interval closure of $P(N)$, denoted often by $[P(N)]$ ).

Then the covering relations $N_{0} \stackrel{P}{\Longrightarrow} N_{1}$ are verified in the simplified version: using interval arithmetic, we simply use a series of ' $<$ ' and ' $>$ ' relations to check if:

- $\left[P\left(N_{0}\right)\right]$ lies in the stripe between the top and bottom borders of $N_{1}$;

- the image of the left part of $N_{0}^{-}$lies to the left of $N_{1}$;

- and the image of the right part of $N_{0}^{-}$lies to the right of $N_{1}$.

The above conditions are sufficient for covering $N_{0} \stackrel{P}{\Longrightarrow} N_{1}$.

We use the CAPD library for C++ (CAPD Group 2017), containing in particular modules for interval arithmetic, linear algebra and Taylor integration.

\section{2 $P_{1}$ and $P_{2}$}

The simplest situation occurs in the case of the points $P_{1}$ and $P_{2}$, because we found a direct topological horseshoe for the first iteration of the Poincaré map $P$ (see Fig. 8).

Theorem 3 Let $N_{0}$ and $N_{1}$ be h-sets of the form $p+A \cdot b \cdot 10^{-3}$, where

\begin{tabular}{llll}
\hline & $p$ & $A$ & $b$ \\
\hline$N_{0}$ & $P_{1}$ & $\mathbf{M}_{1}$ & {$[-0.8,0.8] \times[-180,80]$} \\
$N_{1}$ & $P_{2}$ & $\mathbf{M}_{2}$ & {$[-10,5] \times[-75,200]$} \\
\hline
\end{tabular}

Then the following chain of covering relations occur:

$$
N_{0} \stackrel{P}{\Longrightarrow} N_{0} \stackrel{P}{\Longrightarrow} N_{1} \stackrel{P}{\Longrightarrow} N_{1} \stackrel{P}{\Longrightarrow} N_{0},
$$

which proves the existence of symbolic dynamics for $P$.

Proof Computer assisted (Gierzkiewicz and Zgliczyński 2018). 
Fig. 8 Horseshoe proving symbolic dynamics for $P$, connecting the points $P_{1}$ and $P_{2}$. The exit sets of $h$-sets and their images are marked in orange

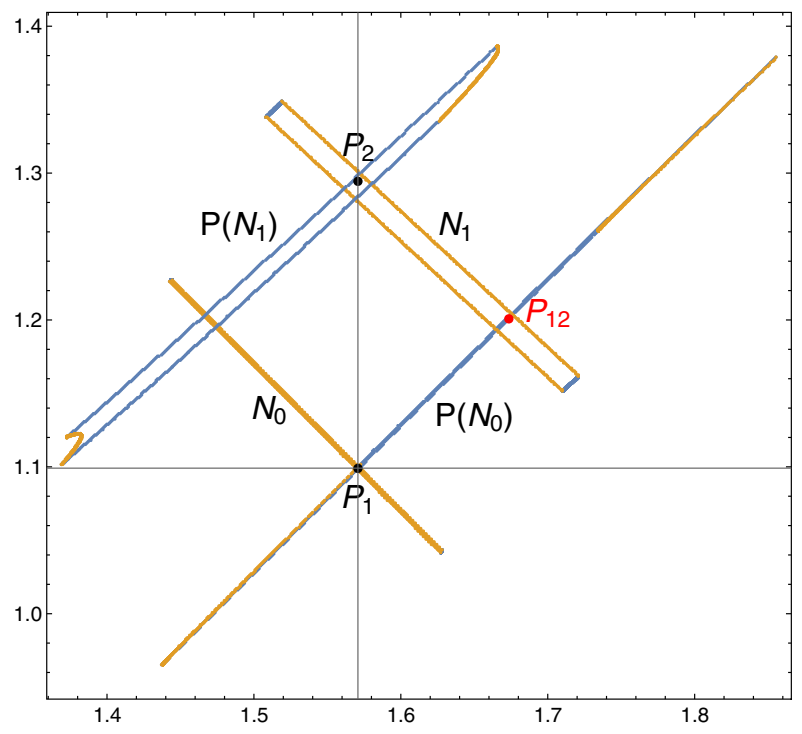

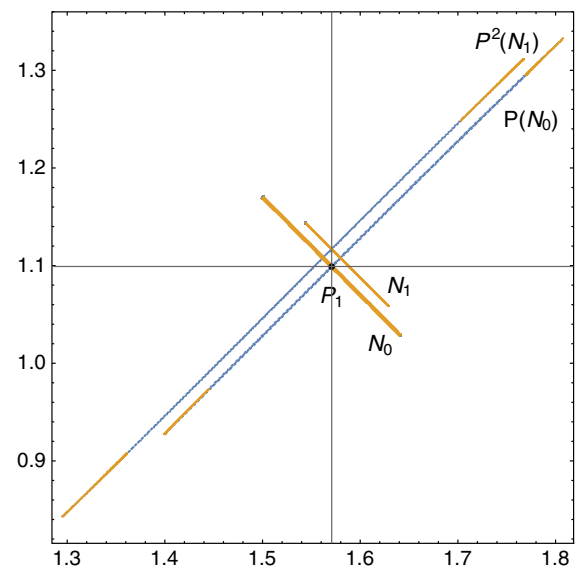

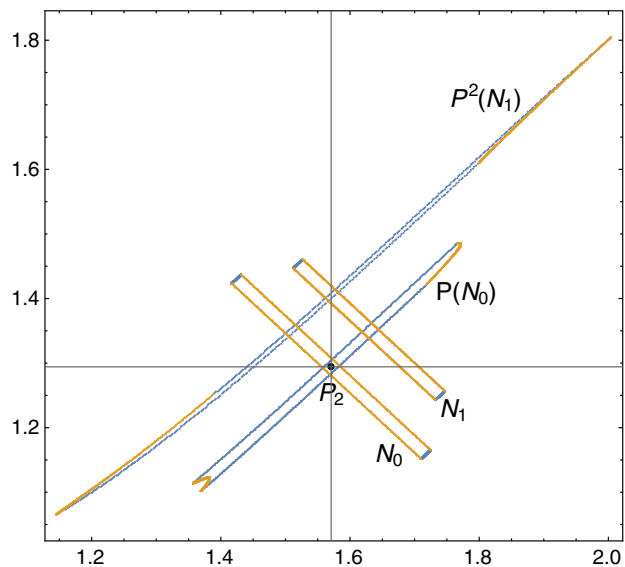

Fig. 9 Horseshoes proving symbolic dynamics for the second iteration of the Poincare map $P^{2}$, connecting the point $P_{1}$ to itself (to the left) or $P_{2}$ to itself (to the right). The exit sets and their images are marked in orange

\section{3 $P_{1}$ and $P_{1}$, and $P_{2}$ and $P_{2}$}

To prove symbolic dynamics between $P_{1}$ and $P_{1}$ or between $P_{2}$ and $P_{2}$ with our simple tools, we need the second iteration of the Poincaré map $P$. Figure 9 illustrates the situation.

Theorem 4 Let $N_{0}$ and $N_{1}$ be h-sets of the form $p+A \cdot b \cdot 10^{-3}$, where 


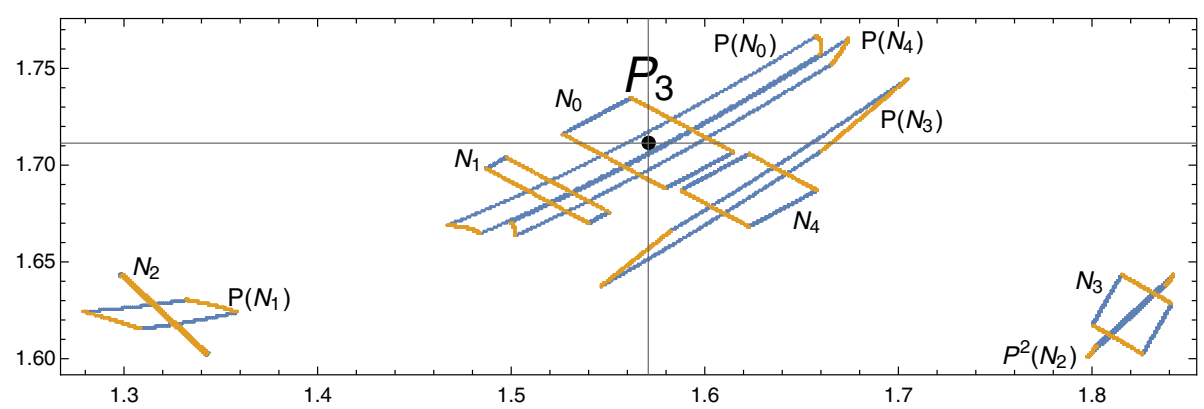

Fig. 10 Sequence of covering relations proving symbolic dynamics for $P^{5}$, connecting the point $P_{3}$ with itself. The exit sets and their images are marked in orange

\begin{tabular}{llll}
\hline & $p$ & $A$ & $b$ \\
\hline$N_{0}$ & $P_{1}$ & $\mathbf{M}_{1}$ & {$[-1,1] \times[-100,100]$} \\
$N_{1}$ & $(1.58669 ; 1.10102)$ & $\mathbf{M}_{1}$ & {$[-0.1,0.1] \times[-60,60]$} \\
\hline
\end{tabular}

or

\begin{tabular}{llll}
\hline & $p$ & $A$ & $b$ \\
\hline$N_{0}$ & $P_{2}$ & $\mathbf{M}_{2}$ & {$[-10,10] \times[-200,200]$} \\
$N_{1}$ & $(1.62953 ; 1.35174)$ & $\mathbf{M}_{2}$ & {$[-10,10] \times[-150,150]$} \\
\hline
\end{tabular}

Then, in both cases we have the following sequence of covering relations:

$$
N_{0} \stackrel{P}{\Longrightarrow} N_{0} \stackrel{P}{\Longrightarrow} N_{1} \stackrel{P^{2}}{\Longrightarrow} N_{1} \stackrel{P^{2}}{\Longrightarrow} N_{0}
$$

which proves the existence of symbolic dynamics for $P^{2}$ (see Remark 1).

Proof Computer assisted (Gierzkiewicz and Zgliczyński 2018).

\section{4 $P_{3}$ and $P_{3}$}

To construct the horseshoe connecting $P_{3}$ to $P_{3}$, we will need five iterations of $P$ (see Fig. 10). Note that for $N_{2}$ and $N_{3}$ the direction matrices are corrected to make them compatible to the dynamics along the stable or unstable manifold.

Theorem 5 Let $N_{i}, i=0, \ldots, 4$ be h-sets of the form $p+A \cdot b \cdot 10^{-3}$, where 


\begin{tabular}{|c|c|c|c|c|}
\hline & $p$ & $A$ & & $b$ \\
\hline $\begin{array}{l}N_{0} \\
N_{1}\end{array}$ & $\begin{array}{l}P_{3} \\
(1.51877 ; 1.68699)\end{array}$ & \multirow{2}{*}{$\begin{array}{l}\mathbf{M}_{3} \\
\mathbf{M}_{3} \\
{\left[\begin{array}{l}0.734429 \\
0.678686\end{array}\right.}\end{array}$} & \multirow[b]{2}{*}{$\left.\begin{array}{c}0.734429 \\
-0.678686\end{array}\right]$} & $\begin{array}{l}{[-20,20] \times[-30,30]} \\
{[-6,6] \times[-30,30]}\end{array}$ \\
\hline$N_{2}$ & $(1.32082 ; 1.62293)$ & & & {$[-0.5,0.5] \times[-30,30]$} \\
\hline$N_{3}$ & $(1.82077 ; 1.62293)$ & \multirow{2}{*}{ 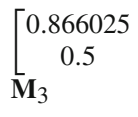 } & \multirow[t]{2}{*}{$\left.\begin{array}{c}-0.5 \\
0.866025\end{array}\right]$} & {$[-15,15] \times[-15,15]$} \\
\hline$N_{4}$ & $(1.62282 ; 1.68699)$ & & & {$[-20,20] \times[-20,20]$} \\
\hline
\end{tabular}
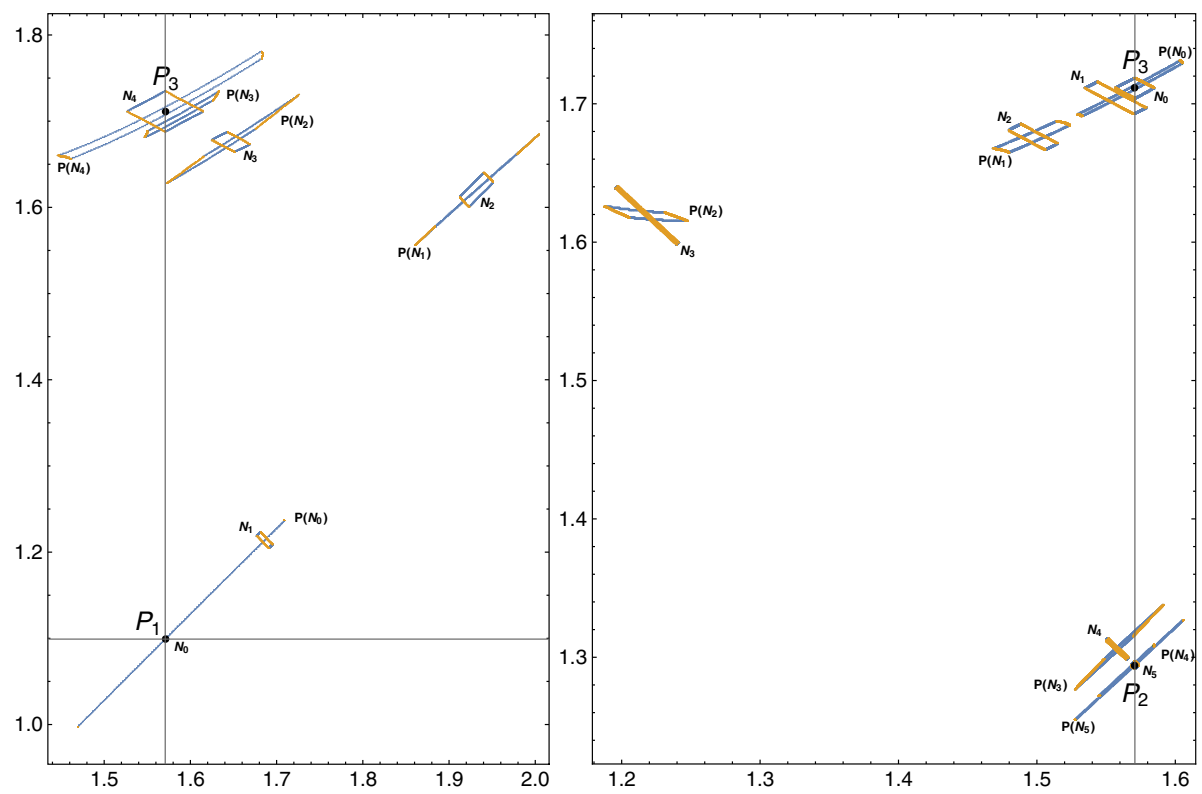

Fig. 11 To the left: the right half of the horseshoe proving symbolic dynamics for $P^{4}$, connecting the points $P_{1}$ and $P_{3}$. To the right: the left half of the horseshoe proving symbolic dynamics for $P^{5}$, connecting the points $P_{3}$ and $P_{2}$. The exit sets and their images are marked in orange

Then we have the following sequence of covering relations:

$$
N_{0} \stackrel{P}{\Longrightarrow} N_{0} \stackrel{P}{\Longrightarrow} N_{1} \stackrel{P}{\Longrightarrow} N_{2} \stackrel{P^{2}}{\Longrightarrow} N_{3} \stackrel{P}{\Longrightarrow} N_{4} \stackrel{P}{\Longrightarrow} N_{0} \text {, and also } N_{4} \stackrel{P}{\Longrightarrow} N_{1},
$$

which proves the existence of symbolic dynamics in $N_{0} \cup N_{1}$ for $P^{5}$ (see Remark 1).

Proof Computer assisted (Gierzkiewicz and Zgliczyński 2018).

\section{5 $P_{1}$ and $P_{3}$, and $P_{2}$ and $P_{3}$}

To connect $P_{1}$ and $P_{3}$, we need the fourth iteration of $P$ (see Fig. 11), but this time we find only a one-way chain of covering relations from $P_{1}$ to $P_{3}$. Thanks to the time-reversing symmetry and the fact that we choose $N_{0}$ and $N_{4}$ symmetrical related to the $\theta=\frac{\pi}{2}$ line, we will be able to close this chain.

Theorem 6 Let $N_{i}, i=0, \ldots, 4$ be h-sets of the form $p+A \cdot b \cdot 10^{-3}$, where 


\begin{tabular}{llll}
\hline & $p$ & $A$ & $b$ \\
\hline$N_{0}$ & $P_{1}$ & $\mathbf{M}_{1}$ & {$[-0.65,0.65] \times[-0.65,0.65]$} \\
$N_{1}$ & $(1.68635 ; 1.21391)$ & $\mathbf{M}_{1}$ & {$[-3,3] \times[-10,10]$} \\
$N_{2}$ & $(1.93186 ; 1.62049)$ & $\mathbf{M}_{1}$ & {$[-20,20] \times[-8,8]$} \\
$N_{3}$ & $(1.6471 ; 1.67581)$ & $\mathbf{M}_{3}$ & {$[-10,10] \times[-15,15]$} \\
$N_{4}$ & $P_{3}$ & $\mathbf{M}_{3}$ & {$[-25,25] \times[-25,25]$} \\
\hline
\end{tabular}

Then the following covering relations occur:

$$
N_{0} \stackrel{P}{\Longrightarrow} N_{0} \stackrel{P}{\Longrightarrow} N_{1} \stackrel{P}{\Longrightarrow} N_{2} \stackrel{P}{\Longrightarrow} N_{3} \stackrel{P}{\Longrightarrow} N_{4} \stackrel{P}{\Longrightarrow} N_{4}
$$

Proof Computer assisted (Gierzkiewicz and Zgliczyński 2018).

Corollary 2 Let $N_{i}, i=0, \ldots, 4$ be h-sets defined in Theorem 6. Then there is symbolic dynamics in $N_{0} \cup N_{4}$ for $P^{4}$.

Proof Consider the $h$-sets $R\left(N_{i}\right)^{\mathrm{T}}, i=0, \ldots, 4$. Using the time-reversing symmetry $R$, it is clear that

$$
R\left(N_{0}\right) \stackrel{P^{-1}}{\Longrightarrow} R\left(N_{1}\right) \stackrel{P^{-1}}{\Longrightarrow} R\left(N_{2}\right) \stackrel{P^{-1}}{\Longrightarrow} R\left(N_{3}\right) \stackrel{P^{-1}}{\Longrightarrow} R\left(N_{4}\right) ;
$$

hence, from the definition of back-covering,

$$
R\left(N_{4}\right)^{\mathrm{T}} \stackrel{P}{\Longleftarrow} R\left(N_{3}\right)^{\mathrm{T}} \stackrel{P}{\Longleftarrow} R\left(N_{2}\right)^{\mathrm{T}} \stackrel{P}{\Longleftarrow} R\left(N_{1}\right)^{\mathrm{T}} \stackrel{P}{\Longleftarrow} R\left(N_{0}\right)^{\mathrm{T}} .
$$

We have chosen $N_{0}$ and $N_{4}$ to be $\left\{\theta=\frac{\pi}{2}\right\}$-line symmetrical, so $R\left(N_{0}\right)^{\mathrm{T}}=N_{0}$ and $R\left(N_{4}\right)^{\mathrm{T}}=N_{4}$. Therefore, we get the full chain of covering and back-covering relations in the form:

$$
N_{0} \stackrel{P}{\Rightarrow} N_{0} \stackrel{P}{\Rightarrow} N_{1} \stackrel{P}{\Rightarrow} N_{2} \stackrel{P}{\Rightarrow} N_{3} \stackrel{P}{\Rightarrow} N_{4} \stackrel{P}{\Rightarrow} N_{4} \stackrel{P}{\models} R\left(N_{3}\right)^{\mathrm{T}} \stackrel{P}{\models} R\left(N_{2}\right)^{\mathrm{T}} \stackrel{P}{\models} R\left(N_{1}\right)^{\mathrm{T}} \stackrel{P}{\models} N_{0},
$$

which proves the existence of symbolic dynamics in $N_{0} \cup N_{4}$ for $P^{4}$, with the use of Remark 1 .

To connect $P_{2}$ and $P_{3}$ with symbolic dynamics, we need the fifth iteration of $P$ and time-reversing symmetry, see Fig. 11 (right) for illustration.

Theorem 7 Let $N_{i}, i=0, \ldots, 5$ be h-sets of the form $p+A \cdot b \cdot 10^{-3}$, where

\begin{tabular}{llll}
\hline & $p$ & $A$ & $b$ \\
\hline$N_{0}$ & $P_{3}$ & $\mathbf{M}_{3}$ & {$[-8,8] \times[-8,8]$} \\
$N_{1}$ & $(1.5569 ; 1.70419)$ & $\mathbf{M}_{3}$ & {$[-5,5] \times[-20,20]$} \\
$N_{2}$ & $(1.49724 ; 1.67606)$ & $\mathbf{M}_{3}$ & {$[-5,5] \times[-15,15]$} \\
$N_{3}$ & $(1.21834 ; 1.61946)$ & $\mathbf{M}_{2}$ & {$[-1,1] \times[-30,30]$} \\
$N_{4}$ & $(1.55807 ; 1.30592)$ & $\mathbf{M}_{2}$ & {$[-1,1] \times[-10,10]$} \\
$N_{5}$ & $P_{2}$ & $\mathbf{M}_{2}$ & {$[-2,2] \times[-2,2]$} \\
\hline
\end{tabular}

Then we have the following sequence of covering relations:

$$
N_{0} \stackrel{P}{\Longrightarrow} N_{0} \stackrel{P}{\Longrightarrow} N_{1} \stackrel{P}{\Longrightarrow} N_{2} \stackrel{P}{\Longrightarrow} N_{3} \stackrel{P}{\Longrightarrow} N_{4} \stackrel{P}{\Longrightarrow} N_{5} \stackrel{P}{\Longrightarrow} N_{5} \text {. }
$$


Proof Computer assisted (Gierzkiewicz and Zgliczyński 2018).

Corollary 3 Let $N_{i}, i=0, \ldots, 5$ be h-sets defined in Theorem 7. Then there is symbolic dynamics in $N_{0} \cup N_{5}$ for $P^{5}$.

Proof Analogously as in the proof of Corollary 2, the lacking $h$-sets on the right half-plane are simply $R\left(N_{i}\right)^{\mathrm{T}}, i=0, \ldots, 5$. Using the time-reversing symmetry and the definition of back-covering,

$$
R\left(N_{5}\right)^{\mathrm{T}} \stackrel{P}{\Longleftarrow} R\left(N_{4}\right)^{\mathrm{T}} \stackrel{P}{\Longleftarrow} R\left(N_{3}\right)^{\mathrm{T}} \stackrel{P}{\Longleftarrow} R\left(N_{2}\right)^{\mathrm{T}} \stackrel{P}{\Longleftarrow} R\left(N_{1}\right)^{\mathrm{T}} \stackrel{P}{\Longleftarrow} R\left(N_{0}\right)^{\mathrm{T}} .
$$

Again, we have chosen $N_{0}$ and $N_{5}$ to be $\left\{\theta=\frac{\pi}{2}\right\}$-line symmetrical, so $R\left(N_{0}\right)^{\mathrm{T}}=N_{0}$ and $R\left(N_{5}\right)^{\mathrm{T}}=N_{5}$. Therefore, we get the full chain of covering relations:

$$
\begin{aligned}
& N_{0} \stackrel{P}{\Rightarrow} N_{1} \stackrel{P}{\Rightarrow} N_{2} \stackrel{P}{\Rightarrow} N_{3} \stackrel{P}{\Rightarrow} N_{4} \stackrel{P}{\Rightarrow} N_{5} \stackrel{P}{\rightleftarrows} R\left(N_{4}\right)^{\mathrm{T}} \stackrel{P}{\models} R\left(N_{3}\right)^{\mathrm{T}} \stackrel{P}{\rightleftarrows} R\left(N_{2}\right)^{\mathrm{T}} \stackrel{P}{\models} R\left(N_{1}\right)^{\mathrm{T}} \stackrel{P}{\rightleftarrows} N_{0}, \\
& \text { and } \quad N_{0} \stackrel{P}{\Rightarrow} N_{0}, \quad N_{5} \stackrel{P}{\Rightarrow} N_{5},
\end{aligned}
$$

which proves the existence of symbolic dynamics in $N_{0} \cup N_{5}$ for $P^{5}$.

\section{Conclusion}

We would like to underline that we proved purely topological symbolic dynamics. Studying the model for its hyperbolicity is our future plan.

As many proofs are based on the interval arithmetic, our investigation is valid for a small interval of parameters, containing the values for Hyperion. The methods can be easily applied to other (but precise) values of $\omega$ and $e$. The more general question for our future work is to explore the relation between the value of parameters and the size and structure of the chaotic region on the section $S$, which could be applied in modelling rotation of other objects of the universe.

Another question is the way of generalizing the model itself. If it cannot describe the tumbling of Hyperion, then the complete set of Euler equations could be investigated. This extends the phase space from 3 to 7 dimensions and requires the use of more complex methods. The other way to make the model more accurate is to consider the impact of Titan on the Saturn-Hyperion system as a correction in the rotation equation (Tarnopolski 2016) or as a change of parameter $e$ to become an independent variable.

\section{Compliance with ethical standards}

Conflict of interest We declare that we do not have any commercial or associative interest that represents a conflict of interest in connection with the paper submitted.

Open Access This article is distributed under the terms of the Creative Commons Attribution 4.0 International License (http://creativecommons.org/licenses/by/4.0/), which permits unrestricted use, distribution, and reproduction in any medium, provided you give appropriate credit to the original author(s) and the source, provide a link to the Creative Commons license, and indicate if changes were made.

\section{References}

Black, G.J., Nicholson, P.D., Thomas, P.C.: Hyperion: Rotational dynamics. Icarus 117, 149-161 (1995). https://doi.org/10.1006/icar.1995.1148 
CAPD Group: Computer assisted proofs in dynamics C++ library ver. 5.0.6 (2017). http://capd.ii.uj.edu.pl

Danby, J.M.A.: Fundamentals of Celestial Mechanics. Macmillan, New York (1962)

Gierzkiewicz, A., Zgliczyński, P.: C++ source code (2018). http://www.ii.uj.edu.pl/ zgliczyn/

Greiner, W.: Classical Mechanics: Systems of Particles and Hamiltonian Dynamics. Classical Theoretical Physics. Springer, Berlin (2009)

Harbison, R.A., Thomas, P.C., Nicholson, P.C.: Rotational modeling of Hyperion. Celest. Mech. Dyn. Astron. 110(1), 1-16 (2011). https://doi.org/10.1007/s10569-011-9337-3

Jay Klavetter, J.: Rotation of Hyperion. II-dynamics. Astron. J. 98, 1855-1874 (1989)

Moore, R.E.: Interval Analysis. Prentice-Hall Series in Automatic Computation. Prentice-Hall, Englewood Cliffs (1966)

Morse, M., Hedlund, G.: Symbolic dynamics. Am. J. Math. 60, 815-866 (1938)

Moser, J.: Stable and Random Motions in Dynamical Systems: With Special Emphasis on Celestial Mechanics (AM-77), revised edn. Princeton University Press, Princeton (1973)

Neumaier, A.: Interval Methods for Systems of Equations. Encyclopedia of Mathematics and its Applications. Cambridge University Press, Cambridge (1991). https://doi.org/10.1017/CBO9780511526473

Tarnopolski, M.: Nonlinear time-series analysis of Hyperion's lightcurves. Astrophys. Space Sci. 357(2), 160 (2015). https://doi.org/10.1007/s10509-015-2379-3

Tarnopolski, M.: Influence of a second satellite on the rotational dynamics of an oblate moon. Celest. Mech. Dyn. Astron. (2016). https://doi.org/10.1007/s10569-016-9719-7

Wilczak, D., Zgliczyński, P.: Heteroclinic connections between periodic orbits in planar restricted circular three-body problem - a computer assisted proof. Commun. Math. Phys. 234(1), 37-75 (2003). https:// doi.org/10.1007/s00220-002-0709-0

Wilczak, D., Zgliczyński, P.: Heteroclinic connections between periodic orbits in planar restricted circular three body problem. Part II. Commun. Math. Phys. 261(2), 547-547 (2006). https://doi.org/10.1007/ s00220-005-1471-X

Wisdom, J., Peale, S.J., Mignard, F.: The chaotic rotation of Hyperion. Icarus 58(2), 137-152 (1984). https:// doi.org/10.1016/0019-1035(84)90032-0

Zgliczyński, P., Gidea, M.: Covering relations for multidimensional dynamical systems. J. Differ. Equ. 202(1), 32-58 (2004). https://doi.org/10.1016/j.jde.2004.03.013

Publisher's Note Springer Nature remains neutral with regard to jurisdictional claims in published maps and institutional affiliations. 